\title{
Surgical management of penile sarcoid in a stallion
}

\author{
Aram SAADI ${ }^{1}$, Saeed AZIZI ${ }^{2 *}$, Amir-Abbas FARSHID ${ }^{3}$ and Alireza YOUSEFI ${ }^{2}$ \\ ${ }^{1}$ Department of Clinical Pathology and Internal Medicine, Faculty of Veterinary Medicine, Urmia University, Urmia \\ 5756151818, Iran \\ ${ }^{2}$ Department of Surgery and Diagnostic Imaging, Faculty of Veterinary Medicine, Urmia University, Urmia \\ 5756151818, Iran \\ ${ }^{3}$ Department of Pathobiology, Faculty of Veterinary Medicine, Urmia University, Urmia 5756151818, Iran
}

\begin{abstract}
This report describes surgical management and breeding implications of a case of penile sarcoid associated with penis laceration in a 4-year-old Kurdish stallion. A large fleshy mass on the distal end of the penis that resulted in urethral meatus deviation and dysuria was detected in a physical examination. No evidence of local extent or metastasis was detected. Under general anaesthesia, the involved distal portion of the penis was removed through partial phallectomy. Histopathological examination of the mass confirmed a fibroblastic sarcoid. Partial phallectomy was successful for management of penile sarcoid and resulted in no postoperative complications or tumour recurrence in long-term follow up; however, successful ejaculation and semen collection have not been achieved.
\end{abstract}

Key words: partial phalectomy, penis, sarcoid, stallion

\author{
J. Equine Sci. \\ Vol. 30, No. 4 \\ pp. 99-104, 2019
}

Common skin tumours occur on the external genitalia in horses $[1,20]$. The penile neoplasms frequently lead to discomfort and may progress to more severe sequelae and even result in death [22]. Squamous cell carcinoma is the most common form of neoplasia affecting the equine penis [22]. Squamous papillomas, melanomas, fibromas, adenocarcinomas, fibrosarcomas, basal cell carcinomas, neurofibromas, lipomas, and haemangiosarcomas are other reported penile tumours $[1,3,20]$. Sarcoids are the most common skin neoplasm, with reported prevalence rates ranging from 12.9 to $67 \%$ [14]. They can be found anywhere on the body singly or in clusters [14]. Sarcoids occasionally affect the external lamina of the prepuce and scrotum; however, the penis is rarely affected [1]. The purpose of the present report is to describe clinical and histopathological features, surgical management, and breeding implications of a single penile sarcoid in a 4-year-old Kurdish stallion.

Case history: A 4-year-old Kurdish stallion was referred to the Veterinary Medicine Teaching Hospital, Urmia

Received: May 9, 2019

Accepted: September 10, 2019

*Corresponding author. e-mail: s.azizi@urmia.ac.ir

(C)2019 Japanese Society of Equine Science

This is an open-access article distributed under the terms of the Creative Commons Attribution Non-Commercial No Derivatives (by-nc-nd) License. (CC-BY-NC-ND 4.0: https://creativecommons.org/licenses/ by-nc-nd/4.0/)
University, Urmia, Iran, for evaluation of a soft tissue mass on the penis. The horse had a history of penile laceration (due to a foreign body) at the site of the glans six months previously, which had been sutured by a local veterinarian using number 1 nylon in a simple interrupted pattern at that time. Complete healing had not been achieved, and serosanguinous exudate had been observed at the wound site after one month. As a conventional treatment, irrigation with dilute povidone iodine solution and a topical antibiotic ointment had been used intermittently to prevent chronic unhealed wound infection. The first symptom of proud flesh was a small tissue bulge noticed by the owner approximately two months after the injury occurred and 4 months prior to referral, with the small tissue bulge gradually enlarging, leading to the penis hanging out. The stallion had previously bred successfully.

Clinical findings: At the time of referral, the stallion was bright, alert, and in good body condition with a body weight of approximately $400 \mathrm{~kg}$. Vital signs including heart rate (32 beats/min), respiratory rate (12 breaths/min), and rectal temperature $\left(38.2^{\circ} \mathrm{C}\right)$ were within their normal ranges. A fleshy nodular mass measuring $11 \times 14 \mathrm{~cm}$ was present on the distal end of the penis (Figs. 1A and 2A). The urinary meatus was distorted and shifted into a left lateral position (Fig. 1B), and urethral stricture was noted that resulted in dysuria. No other lesions were identified on the prepuce or other parts of the body on physical examination. The gross 
tumour margins were determined via ultrasonographic examination, and lesion extension to the proximal portion of the mass was not detected. There was no evidence of metastasis to the abdomen, thorax, or other distant sites following regional lymph nodes assessment, thoracic radiography, and rectal examination. The haematological and biochemical profiles as well as the results of a urine analysis were within their normal ranges.

Differential diagnosis: Based on a macroscopic evaluation of the mass, the principle differential diagnoses included fibroblastic sarcoids, exuberant granulation tissue, squamous cell carcinomas, infectious granulomas, and habronemiasis.

Treatment: In view of the glans and urinary meatus destruction, dysuria, the large size of the mass, the penis distal end involvement with no proximal spreading, no involvement of the prepuce, and no evidence of metastasis, it was decided that complete surgical excision should be performed (partial phallectomy). Feed was withheld for 12 $\mathrm{hr}$ prior to surgery. The horse received penicillin $\mathrm{G} /$ procaine $(20,000 \mathrm{IU} / \mathrm{kg}, \mathrm{IM})$ and gentamicin sulfate $(2.20 \mathrm{mg} / \mathrm{kg}$, IV Aburaihan Pharmaceutical Co., Tehran, Iran) as pre-surgical antimicrobial agents before anaesthesia induction. A combination of xylazine hydrochloride (Xylazine, Alfasan, Woerden, Netherlands; $0.20 \mathrm{mg} / \mathrm{kg}$, IV) and acepromazine maleate (Neurotranq, Alfasan, Woerden, Netherlands; 0.020 $\mathrm{mg} / \mathrm{kg}$, IV) was administered as pre-medication. After 12 min, the animal was restrained on a surgical table, and $10 \%$ thiopental sodium (Pentothal ${ }^{\circledR}$ Ceva Laboratories, France, $7 \mathrm{mg} / \mathrm{kg} \mathrm{IV}$ ) was used for general anaesthesia induction. Following endotracheal intubation, anaesthesia was maintained with isoflurane $(1.50-2 \%)$ in oxygen (AErrance ${ }^{\circledR}$, Baxter Healthcare, Puerto Rico). The horse was positioned in dorsal recumbency, and the penis was aseptically prepared and draped for surgery. Normal saline $(10 \mathrm{ml} / \mathrm{kg} / \mathrm{hr})$ was administered via a jugular vein catheter during general anaesthesia to help maintain cardiovascular function. A urethral catheter was passed to guide the cutting process, and a tourniquet was applied to the proximal part of the proposed amputation site of the penis as a method to control haemorrhage.

Williams' technique of phallectomy [16] was adopted with a safety margin of at least $1 \mathrm{~cm}$. In brief, a triangular area was removed from the penis ventrum. The apex of the triangular area was directed proximally. The urethra was split along its midline from the base to the apex of the triangular area with blind and sharp cuts. The edges of the urethra and the epithelial edges of the triangular area were opposed using 2-0 Vicryl in simple continuous sutures incorporating and compressing the cavernous tissue of the corpus spongiosum penis. Moreover, the transected edge of the corpus spongiosum penis at the base of the triangular area was compressed using 2-0 Vicryl in a simple continuous suture line through the urethral mucosa and tunica albuginea. The penis was transected obliquely at the base of the triangular urethrostomy in a craniodorsal direction after urethral catheter removal (Fig. 2B). The external pudendal vessel branches located in the dorsal and lateral aspects of the tunica albuginea were ligated with 2-0 Dexon sutures. The stump, including the corpus cavernosum penis, was closed with interrupted simple sutures at predetermined equidistant intervals with 0 Nylon passing through the urethra, tunica albuginea of the urethral groove and tunica albuginea of the dorsum of the corpus cavernosum penis, and penile epithelium (Fig. 2C).

Post-operative care: The horse received penicillin G/ procaine (20,000 IU/kg, IM q12hr), gentamicin sulfate (2.20 $\mathrm{mg} / \mathrm{kg}$, IV q8hr; Aburaihan Pharmaceutical Co., Tehran, Iran $)$, and flunixin meglumine $(0.50 \mathrm{mg} / \mathrm{kg}$, IV q12hr; Razak Pharmaceutical Co., Tehran, Iran) for 5 consecutive days after surgery. Hand walking was allowed. In the short-term follow-up, mild haemorrhage at the end of urination along with preputial oedema was observed for 5 days after surgery which resolved without further treatment. No signs of suture dehiscence or tissue inflammation were seen at the surgical site.

Histopathological findings: The gross appearance was that of a tumorous, nodular, firm, fleshy mass with a uniform structure (Fig. 2D). Standard tissue samples were harvested from the excised mass. After fixation in 10\% neutral-buffered formalin, the samples were embedded in paraffin, and $5 \mu \mathrm{m}$ sections were cut and stained with haematoxylin and eosin. Light microscopy mainly revealed epidermal hyperplasia and rete peg (Fig. 3A) and picket fence formations. Fibroblast proliferations with various amounts of collagen in the dermis (Fig. 3B) as well as a mild degree of hyperkeratosis were also observed. Focal cellular infiltrations (Fig. 3C) and cystic sebaceous glands (Fig. 3D) were found in some areas. These changes are consistent with fibroblastic sarcoid [17].

Outcome: In the long-term (3.5 years) follow-up, no complications, including urine-induced dermatitis, urethral stricture, dysuria, and cystitis, were noticed, and there was no evidence of tumour recurrence at the site of amputation. Two years after operation, based on the owner's request, the horse was used for breeding purposes. Due to the short amputated penis and lack of the glans, the horse was unsuccessful in natural breeding and intromission; however, he had normal sexual behavior and successful erection. The impossibility of natural breeding service and/or semen collection using an artificial vagina or manual stimulation was informed before the operation. Hence, pharmacologically induced ejaculation was performed based on both the Johnston and DeLuca (1998) and McDonnell (2001) protocols. Accordingly, imipramine hydrochloride $(2-3 \mathrm{mg} / \mathrm{kg}$, 


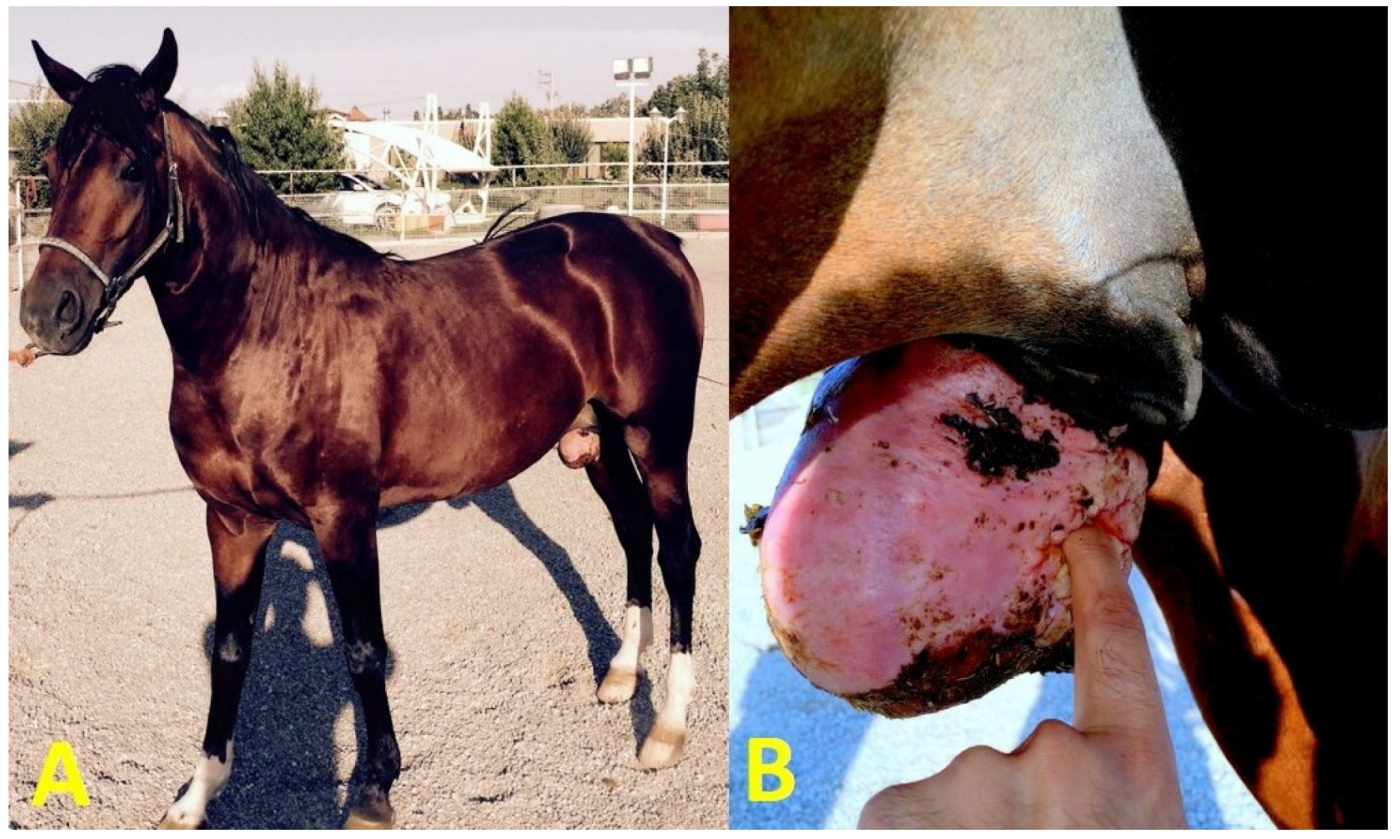

Fig. 1. (A) Single penile sarcoid in a 4-year-old Kurdish stallion. (B) Urethral meatus deviation (finger).

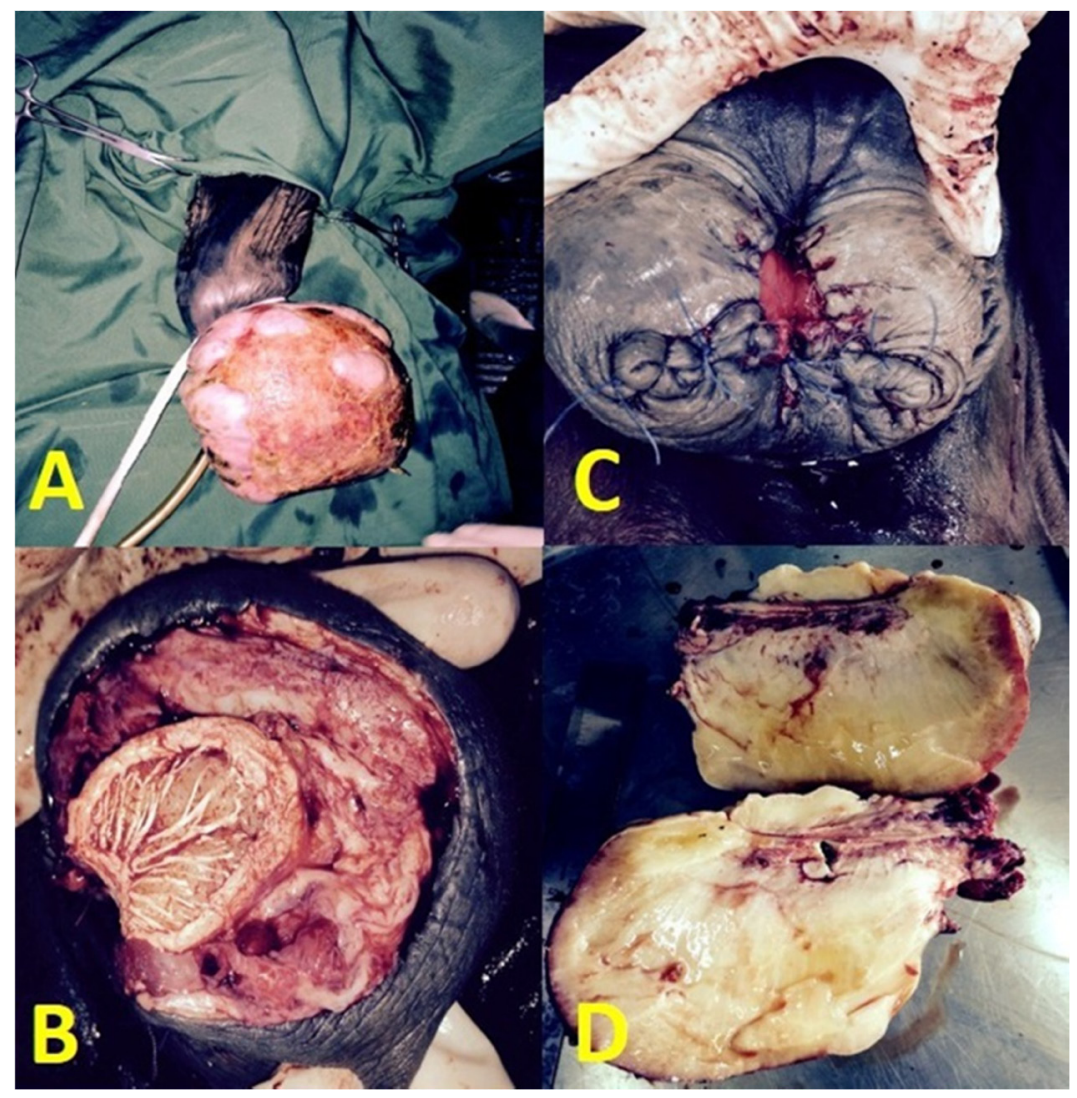

Fig. 2. (A) Fibroblastic nodular appearance of the soft tissue mass of the penile sarcoid. (B) Cross section of the penis stump after partial phallectomy. (C) Final appearance of the penile stump and the new surgically created urethral opening. (D) Cross section of the surgically excised tumour mass. 


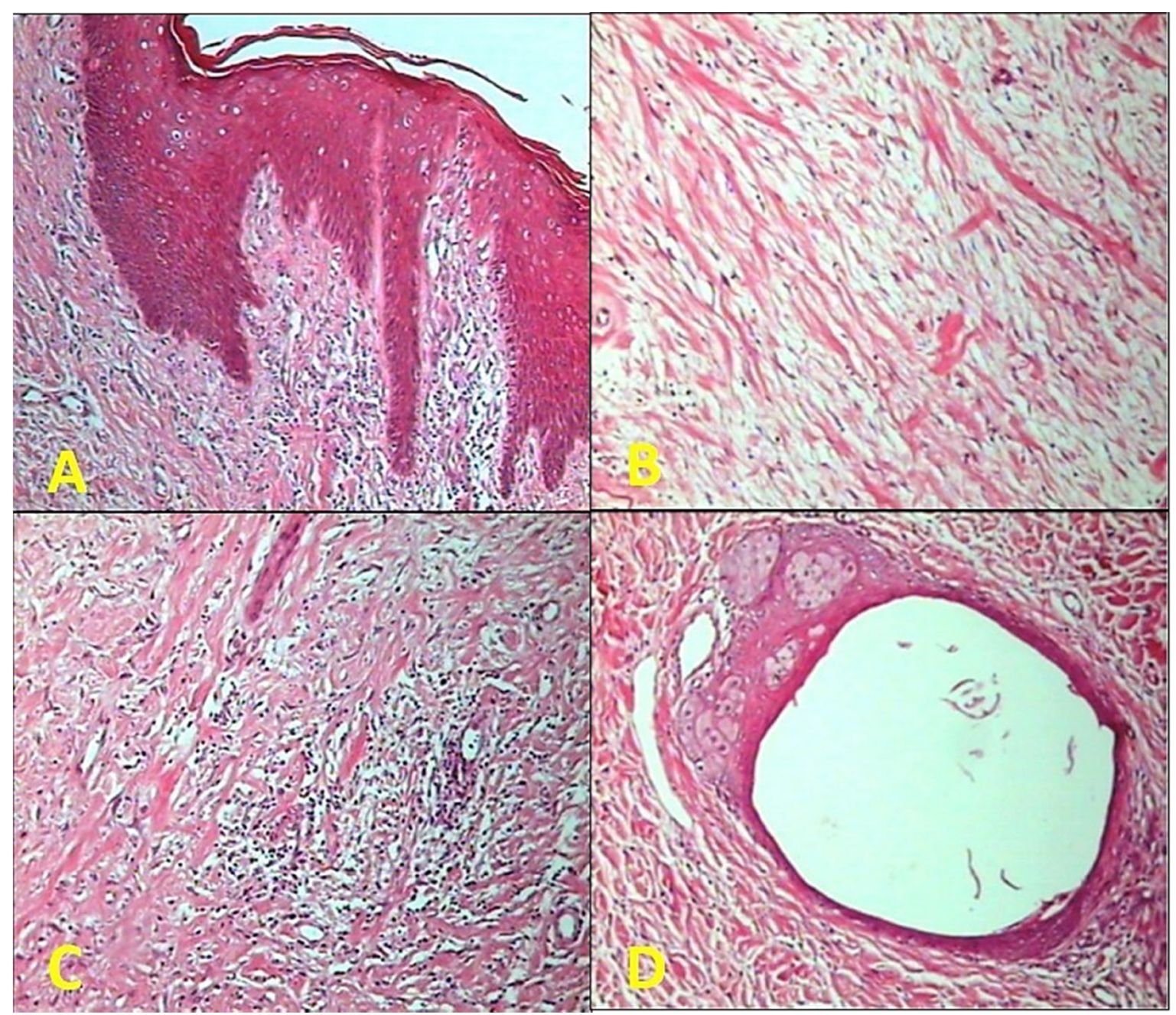

Fig. 3. Photomicrographs of fibroblastic penile sarcoid. (A) Epidermal hyperplasia and rete pegs deep into the dermis. (B) Dermal fibroblast proliferation with collagen. (C) Dermal focal cellular infiltration. (D) Cystic sebaceous gland. Haematoxylin and eosin, $\times 100$.

PO) was administered $1-2$ hr before xylazine hydrochloride $(0.3-0.7 \mathrm{mg} / \mathrm{kg}$, IV) administration on six days in repeated attempts and with titration of the drug dose in the presence or absence of an estrous mare. Despite full erection, sexual arousal, and pre-ejaculatory fluid, ejaculation did not occur.

Discussion: External genitalia neoplasms are not uncommon in horses and can cause discomfort as well as serious complications. The prepuce and penis are covered with skin and a mucosa and thus can be affected by tumours of epithelial and/or mesenchymal origin [21]. Squamous cell carcinoma is the most common tumour of the equine penis and prepuce and is mainly found in aged animals [11, 20]. Papillomas are also common on the external genitalia of male horses. There is often more than one, and they can be present anywhere on the penis [20, 22]. Melanocytic tumours have also been reported to affect all parts of the penis and prepuce other than the glans $[19,20]$. Other tumour types are uncommon findings on the external genitalia of male horses, particularly the penis. The prevalence of other types of penile tumours including haemangiosarcoma [3], neurofibrosarcoma, fibrosarcomas, adenocarcinomas, basal cell carcinomas [20], fibropapilloma [8], and lymphosarcoma [11] was estimated to be $0.03-0.14 \%$ [22]. Sarcoids occasionally affect the external genitalia, but they are more commonly found on the scrotum and prepuce. The penis is rarely affected by sarcoids [1], and penile sarcoid has rarely been reported in the horse [7] or donkey [15]; in both these animals, which little is known about its diagnosis, prognosis, and treatment.

Equine sarcoids (ESs), as the most common neoplasm in horses, are locally invasive, non-metastatic tumours that rarely regress spontaneously. Sex, coat colour, and seasonal and geographical predilections have not been identified; however, the majority of the reported affected animals were 
1-7 years old [14]. ESs can occur on any part of the body, either singly or in clusters including the ventral abdomen, limbs, and head, either singly or in clusters. Specifically, around the eyes, pinnae, and commissures of the lips are the most commonly affected sites [14]. Six types of sarcoid with varying treatment recommendations and prognoses have been recognized, including occult, veracious, nodular, fibroblastic, malevolent, and mixed sarcoids. In the present report, the affected horse had a single raised fleshy fibroblastic sarcoid (proud flesh-like) only on the penile tissue and had a history of trauma at the site of a previous laceration (6 months previously). Skin wound sites, regardless of location, as well as sites of other sarcoid types subjected to trauma are predilection sites for fibroblastic sarcoid [17].

Although it does not metastasize, the sarcoid is not considered a benign neoplasm and can provide significant therapeutic challenges, whether surgical or medical managements are required [14]. In general, the choice and success of sarcoid therapy are dependent on factors such as site, size, aggressiveness and number of lesions, previous attempts at treatment, clinician experience, and availability of services, equipment and facilities [17]. On the other hand, since little is known about sarcoid behavior on the equine penis, penile sarcoid poses significant treatment challenges; however, the ideal approach for treatment of equine penile tumours is surgical excision with preservation of external genitalia functions including micturition, erection, and ejaculation [22].

In this case, due to the presence of a large mass at the distal end of the penis without proximal spread and metastasis, urethral stricture, and dysuria, partial phallectomy was performed to remove the tumour mass and alleviate the horse's discomfort. Under the same circumstances, partial phallectomy has been recommended as an ideal approach to treat penile masses such as tumours [22] and habronemiasis [18]. Williams' technique of phallectomy was chosen in this case due to a lower risk of post-operative urethral stricture and urine scalding [16]. In this stallion, short-term complications in the immediate post-operative period ( 5 days) included mild preputial oedema and haemorrhage at the end of urination which resolved without further treatments as has been described in previous reports $[6,10]$. In the long-term follow-up, there was also no gross evidence of recurrence. It has been reported that the recurrence rate of ESs varies between 15 and $82 \%$, with most tumours recurring, often more aggressively, within 6 months [14]; however, surgical removal with an included safety margin of at least one centimetre has been recommended to prevent tumour recurrence [12].

In the stallion, preservation of breeding function may not be possible following partial phallectomy [3]. In line with this, castration has been recommended 3-4 weeks before penile amputation to minimize complications associated with sexual excitation and erection, namely post-operative haemorrhage and dehiscence of the suture lines [16]; however, in the present case, the owner hoped to preserve the horse's fertility and was not prepared to allow the stallion to be castrated. Clearly, natural mating and ejaculation induction using an artificial vagina or manual stimulation was not possible due to the lack of the glans [4]. Pharmacological methods for enhancing and inducing ejaculation in stallions with ejaculatory dysfunctions or disabilities interfering with natural breeding function have been described previously [13]. Nonetheless, chemically induced ejaculation using imipramine orally followed by xylazine intravenously $[9,13]$ was not successful in this case. The success rate of ejaculation was estimated by Johnston and Deluca to be about 56\% [9], and McDonnell estimated it to be about $67 \%$ [13], though titration of the dose in individual stallions significantly improves the ejaculation rate for any given treatment [13]. Electroejaculation, as an alternative method, is a feasible method of semen collection from stallions with some of the physical and psychological problems. Its use in the horse has been demonstrated but not practiced due to the relatively high voltages required for electro-ejaculation to be achieved [5]. Harvesting of epididymal sperm from stallions for freezing and future use is generally considered a method of last resort to preserve valuable genetic material when stallions suffer a catastrophic injury or die prematurely. It is also sometimes requested at the time of elective castration [2].

In summary, partial phallectomy using Williams technique was an effective surgical method for treatment of solitary fibroblastic sarcoid confined to the distal end, or glans, of the penis in the horse. Though normal precopulatory behavior, erection, and mounting persisted, ejaculation was ultimately unsuccessful in the horses after partial phallectomy.

\section{References}

1. Brinsko, S.P. 1998. Neoplasia of the male reproductive tract. Vet. Clin. North Am. Equine Pract. 14: 517-533. [Medline] [CrossRef]

2. Brinsko, S.P. 2011. Semen collection techniques and insemination procedures. pp. 1268-1277. In: Equine Reproduction, 2nd ed. (McKinnon, A.O., and Squires, E.L. eds.), John Wiley \& Sons, Ames.

3. Byrne, D.P., Woolford, L., and Booth, T.M. 2014. Penile haemangiosarcoma in a breeding stallion. Equine Vet. Educ. 28: 304-309. [CrossRef]

4. Crump, J. Jr., and Crump, J. 1989. Stallion ejaculation induced by manual stimulation of the penis. Theriogenology 31: 341-346. [Medline] [CrossRef]

5. Davies Morel, M.C.G. 1999. Equine Artificial Insemina- 
tion. pp. 151-189. CABI Pub., New York.

6. Doles, J., Williams, J.W., and Yarbrough, T.B. 2001. Penile amputation and sheath ablation in the horse. Vet. Surg. 30: 327-331. [Medline] [CrossRef]

7. Farjani-Kish, G., Dehghani-Nazhvani, S., Namazi, F., and Jalali, Y. 2014. Penile sarcoid in horse: clinicopathological characteristics with brief literature review. Comp. Clin. Pathol. 23: 459-462. [CrossRef]

8. Gardiner, D.W., Teifke, J.P., Podell, B.K., and Kamstock, D.A. 2008. Fibropapilloma of the glans penis in a horse. $J$. Vet. Diagn. Invest. 20: 816-819. [Medline] [CrossRef]

9. Johnston, P.F., and Deluca, J.L. 1998. Chemical ejaculation of stallions after administration of oral imipramine followed by intravenous xylazine. Proc. Am. Assoc. Equine Pract. 43: 59-62.

10. Mair, T.S., Walmsley, J.P., and Phillips, T.J. 2000. Surgical treatment of 45 horses affected by squamous cell carcinoma of the penis and prepuce. Equine Vet. J. 32: 406-410. [Medline] [CrossRef]

11. Markel, M.D., Wheat, J.D., and Jones, K. 1988. Genital neoplasms treated by en bloc resection and penile retroversion in horses: 10 cases (1977-1986). J. Am. Vet. Med. Assoc. 192: 396-400. [Medline]

12. Martens, A., De Moor, A., Vlaminck, L., Pille, F., and Steenhaut, M. 2001. Evaluation of excision, cryosurgery and local BCG vaccination for the treatment of equine sarcoids. Vet. Rec. 149: 665-669. [Medline] [CrossRef]

13. McDonnell, S.M. 2001. Oral imipramine and intravenous xylazine for pharmacologically-induced ex copula ejaculation in stallions. Anim. Reprod. Sci. 68: 153-159. [Medline] [CrossRef]

14. Rashmir-Raven, A.M. 2018. Disorders of the skin. pp. 1159-1216. In: Equine Internal Medicine, 4th ed. (Reed,
S.M., Bayly, W.M., and Sellon, D.C. eds.), Elsevier, St. Louis.

15. Rizk, A., Mosbah, E., Karrouf, G., and Abou Alsoud, M. 2013. Surgical management of penile and preputial neoplasms in equine with special reference to partial phallectomy. J. Vet. Med. 2013: 891413. [Medline] [CrossRef]

16. Schumacherm, J. 2012. Penis and prepuce. pp. 840-865. In: Equine Surgery, 4th ed. (Auer, J.A. and Stick, J.A. eds.), Elsevier, St. Louis.

17. Scott, D.W., and Miller, W.H. Jr. 2011. Equine Dermatology, 2nd ed. pp. 468-516. Saunders, St. Louis.

18. Tyrnenopoulou, P., Diakakis, N., Psalla, D., Traversa, D., Papadopoulos, E., and Antonakakis, M. 2019. Successful surgical management of eosinophilic granuloma on the urethral process of a gelding associated with Habronema spp. infection. Equine Vet. Educ. 31: e1-e4. [CrossRef]

19. Valentine, B.A. 1995. Equine melanocytic tumors: a retrospective study of 53 horses (1988 to 1991). J. Vet. Intern. Med. 9: 291-297. [Medline] [CrossRef]

20. van den Top, J.G.B., de Heer, N., Klein, W.R., and Ensink, J.M. 2008. Penile and preputial tumours in the horse: a retrospective study of 114 affected horses. Equine Vet. J. 40: 528-532. [Medline] [CrossRef]

21. van den Top, J.G.B., Ensink, J.M., Barneveld, A., and Van Weeren, P.R. 2011. Penile and preputial squamous cell carcinoma in the horse and proposal of a classification system. Equine Vet. Educ. 23: 636-648. [CrossRef]

22. van den Top, J.G.B., Ensink, J.M., Gröne, A., Klein, W.R., Barneveld, A., and Van Weeren, P.R. 2010. Penile and preputial tumours in the horse: literature review and proposal of a standardised approach. Equine Vet. J. 42: 746-757. [Medline] [CrossRef] 